\title{
Attenuation of brain edema and spatial learning deficits by the inhibition of NADPH oxidase activity using apocynin following diffuse traumatic brain injury in rats
}

\author{
SI-XIN SONG ${ }^{1}$, JUN-LING GAO ${ }^{2}$, KAI-JIE WANG ${ }^{3}$, RAN LI $^{2}$, YAN-XIA TIAN ${ }^{2}$, \\ JIAN-QIANG WEI $^{1}$ and JIAN-ZHONG CUI ${ }^{1,3}$ \\ ${ }^{1}$ Department of Surgery, Hebei Medical University, Shijiazhuang 050017; \\ ${ }^{2}$ School of Basic Medical Sciences, Hebei United University; \\ ${ }^{3}$ Tangshan Workers' Hospital, Tangshan 063000, P.R. China
}

Received June 1, 2012; Accepted October 12, 2012

DOI: $10.3892 / \mathrm{mmr} .2012 .1147$

\begin{abstract}
Diffuse brain injury (DBI) is a leading cause of mortality and disability among young individuals and adults worldwide. In specific cases, DBI is associated with permanent spatial learning dysfunction and motor deficits due to primary and secondary brain damage. Nicotinamide adenine dinucleotide phosphate (NADPH) oxidase (NOX) is a major complex that produces reactive oxygen species (ROS) during the ischemic period. The complex aggravates brain damage and cell death following ischemia/reperfusion injury; however, its role in DBI remains unclear. The present study aimed to investigate the hypothesis that levels of $\mathrm{NOX}_{2}$ (a catalytic subunit of NOX) protein expression and the activation of NOX are enhanced following DBI induction in rats and are involved in aggravating secondary brain damage. A rat model of DBI was created using a modified weight-drop device. Our results demonstrated that $\mathrm{NOX}_{2}$ protein expression and NOX activity were enhanced in the CA1 subfield of the hippocampus at 48 and $72 \mathrm{~h}$ following DBI induction. Treatment with apocynin (50 mg/kg body weight), a specific inhibitor of NOX, injected intraperitoneally $30 \mathrm{~min}$ prior to DBI significantly attenuated $\mathrm{NOX}_{2}$ protein expression and NOX activation. Moreover, treatment with apocynin reduced brain edema and improved spatial learning function assessed using the Morris water maze. These results reveal that treatment with apocynin may provide a new
\end{abstract}

Correspondence to: Dr Jian-Zhong Cui, Department of Surgery, Hebei Medical University, 361 East Zhongshan Road, Shijiazhuang 050017, P.R. China

E-mail: jzhcuidr2001@163.com

Abbreviations: DMSO, dimethyl sulphoxide; DBI, diffuse brain injury; NOX, nicotinamide adenine dinucleotide phosphate oxidase; ROS, reactive oxygen species

Key words: diffuse brain injury, nicotinamide adenine dinucleotide phosphate oxidase, $\mathrm{NOX}_{2}$, secondary brain damage, learning and memory ability neuroprotective therapeutic strategy against DBI by diminishing the upregulation of $\mathrm{NOX}_{2}$ protein and NOX activity.

\section{Introduction}

Diffuse brain injury (DBI) is a leading cause of mortality and disability in young individuals. The primary damage in DBI is thought to result from mechanical forces applied to the skull and brain at the time of impact, leading to focal or diffuse brain injury patterns. In comparison, secondary brain damage following DBI evolves progressively and is characterized by a complex cascade of biochemical events that cause brain edema and neuronal death and aggravate neurological dysfunctions, including learning and memory deficits.

Nicotinamide adenine dinucleotide phosphate (NADPH) oxidase (NOX) is a multi-subunit enzyme complex localized to the plasma membrane of cells and is a major source of reactive oxygen species (ROS). This enzyme is expressed in neurons, astrocytes and microglia (1-3). There are several subunits of NOX, termed $\mathrm{NOX}_{1-5}(1,4) . \mathrm{NOX}_{2}$ is a catalytic subunit of NOX. A previous study demonstrated that $\mathrm{NOX}_{2}$ is localized to the cerebral cortex and hippocampal CA1 subregion (5). Limited knowledge is currently available on the role of $\mathrm{NOX}_{2}$ in DBI and it remains unclear whether alterations in $\mathrm{NOX}_{2}$ expression and NOX activity are associated with secondary damage following DBI in rats.

Apocynin was first described by Schmiedeberg in 1883 and was isolated from the roots of Apocynum cannabinum (Canadian hemp). Apocynin has since been utilized in a number of experimental models as a NOX complex inhibitor $(6,7)$, however, the mechanism of inhibition of NOX is not well understood in rats following DBI.

In the present study, alterations in $\mathrm{NOX}_{2}$ protein expression and NOX activity in the CA1 subregion of the hippocampus following DBI induction were investigated. In addition, we aimed to determine whether pre-treatment with apocynin results in the attenuation of brain edema and improves spatial cognitive functions by modulation of $\mathrm{NOX}_{2}$ protein expression and NOX activation following DBI induction. The results of the present study may generate insight into the efficacy of 
apocynin against secondary damage following DBI induction, through the inhibition of $\mathrm{NOX}_{2}$ expression and NOX activity.

\section{Materials and methods}

Animals and DBI model. All experimental procedures were performed in accordance with the guidelines of the Chinese Council on Animal Protection and were approved by the Hebei Medical University Committee for the use of animals in research. A total of 140 male Sprague-Dawley rats (age, 12-16 weeks; weight, 350-375 g; Tangshan, China) were used in the present study. All animals were housed with a standard $12 \mathrm{~h}$ light/dark cycle and free access to water and food prior to and following surgery or sham surgery. The rat model of DBI was created using a modified weight-drop device, as described previously by Sawauchi et al (8). Briefly, rats were anesthetized with sodium pentobarbial (Nembutal, $60 \mathrm{mg} / \mathrm{kg}$ ). Under anaesthesia, a midline incision was performed to expose the skull the between bregma and lambda suture lines and a steel disk (diameter, $10 \mathrm{~mm}$; thickness, $3 \mathrm{~mm}$ ) was adhered to the skull using dental acrylic. Animals were placed on a foam mattress underneath a weight-drop device in which a 450-g weight falls freely through a vertical tube from $1.5 \mathrm{~m}$ onto the steel disk. Sham-operated animals underwent the same surgical procedure without weightdrop impact. Rats were housed in individual cages following surgery and placed on heat pads $\left(37^{\circ} \mathrm{C}\right)$ for $24 \mathrm{~h}$ to maintain normal body temperature during the recovery period.

Group and drug administration. Rats were randomly divided into 4 groups: sham, DBI untreated, DBI treated with vehicle (DMSO) and DBI treated with apocynin groups. Apocynin (50 mg/kg body mass) $(9,10)$ and the vehicle were administered by intraperitoneal injection $30 \mathrm{~min}$ prior to sham surgery or DBI induction (11).

Western blot analysis of $\mathrm{NOX}_{2}$ protein expression. At 48 and $72 \mathrm{~h}$ following DBI induction, rats were anesthetized and underwent intracardiac perfusion with $0.1 \mathrm{~mol} / \mathrm{l}$ phosphatebuffered saline ( $\mathrm{pH}$ 7.4). Hippocampal CA1 subregions were rapidly isolated, total proteins were extracted and protein concentration was determined by the BCA reagent (Solarbio, Beijing, China) method. Equal amounts (50 $\mu \mathrm{g})$ of protein were subjected to $10 \%$ SDS-PAGE and electrotransferred onto a hydrophobic PVDF membrane (Roche Diagnostics, Mannheim, Germany). Following blocking, the membrane was incubated overnight at $4^{\circ} \mathrm{C}$ with primary antibodies against $\operatorname{NOX}_{2}(1: 200)$ and $\beta$-actin (1:200; both purchased from Santa Cruz Biotechnology; Santa Cruz, CA, USA). Following incubation with a titrated secondary antibody (1:2,000; Cell Signaling Technology, Inc., Danvers, MA, USA), the immunoblot on the membrane was visualized by development with an enhanced chemiluminescence detection system and densitometric signals were quantified using an imaging program. Immunoreactive bands were normalized to intensity of corresponding bands for $\beta$-actin. Results were analyzed using the National Institutes of Health Image 1.41 software (Bethesda, MD, USA).

NOX activity assay. NOX activity was determined by a colorimetric method $(12,13)$ based on changes in NADPH consumption monitored by the decrease in absorbance at $\lambda=340 \mathrm{~nm}$ in the presence of DPI. Hippocampal CA1 tissue samples collected at 48 and $72 \mathrm{~h}$ following DBI induction were homogenized in Krebs-Ringer phosphate buffer at $\mathrm{pH} 7.4$. Homogenates were centrifuged at $1,000 \mathrm{x}$ g for $10 \mathrm{~min}$ at $4^{\circ} \mathrm{C}$ and the pellets were discarded. Supernatants were spun at $13,000 \mathrm{x} \mathrm{g}$ in an ultracentrifuge for $20 \mathrm{~min}$ at $4^{\circ} \mathrm{C}$ and membrane fractions were separated. Enzyme assays were performed in a final volume of $1 \mathrm{ml}$ containing $50 \mathrm{mM}$ Krebs-Ringer phosphate buffer ( $\mathrm{pH}$ 7.0), $1 \mathrm{mM}$ EGTA, $150 \mathrm{mM}$ sucrose, $0.5 \mathrm{mM}$ lucigenin, $0.1 \mathrm{mM}$ NADPH solution and $50 \mu \mathrm{g}$ membrane fractions. Photoemissions, expressed in terms of relative light units (RLU), were recorded every $1 \mathrm{~min}$ continuously for $5 \mathrm{~min}$ by a standard luminometer. All values were standardized to the amount of protein and were calculated as RLU/ $\mu \mathrm{g} /$ minute.

Evaluation of brain edema. Brain edema was evaluated by analysis of brain water content with the wet-dry weight method as described previously (14). Following this, animals were sacrificed by decapitation under anesthesia at 48 and $72 \mathrm{~h}$ following DBI induction or sham surgery. Brains were separated and weighed immediately to obtain wet weight and dried in a desiccating oven for $24 \mathrm{~h}$ at $100^{\circ} \mathrm{C}$. Dry tissues were weighed again. The percentage of water in the tissues was calculated according to the formula: $\%$ brain water $=[$ (wet weight - dry weight $) /$ wet weight] x100.

Assessment of the spatial learning ability using the Morris water maze. Spatial learning ability was assessed using a Morris water maze as described previously (15). The maze consists of a black circular pool (diameter, $180 \mathrm{~cm}$; height, $45 \mathrm{~cm}$ ) filled with water (depth, $30 \mathrm{~cm}$ ) at $26^{\circ} \mathrm{C}$ and virtually divided into 4 equivalent quadrants: north $(\mathrm{N})$, west $(\mathrm{W})$, south (S) and east (E). A 2-cm submerged escape platform (diameter, $12 \mathrm{~cm}$; height, $28 \mathrm{~cm}$; made opaque with paint) was placed in the center of one of the quadrants, equidistant from the sidewall and the center of the pool. Rats were trained to find the platform prior to DBI or sham surgery. For each trial, the rat was randomly placed into a quadrant start point $(\mathrm{N}, \mathrm{S}, \mathrm{E}$ or $\mathrm{W}$ ) facing the wall of the pool and allowed a maximum of $60 \mathrm{sec}$ to escape to a platform, rats which failed to escape within $90 \mathrm{sec}$ were placed on the platform for a maximum of $20 \mathrm{sec}$ and returned to the cage for a new trial (intertrial interval $20 \mathrm{sec}$ ). Maze performance was recorded by a video camera suspended above the maze and interfaced with a video tracking system (HVS Imaging, Hampton, UK). The average escape latency of a total of five trials was calculated. Tests were conducted $72 \mathrm{~h}$ following DBI induction.

Statistical analysis. Data are expressed as the means \pm standard error. Statistical analysis was performed using ANOVA and followed by the Student-Newman-Keuls post-hoc test. $\mathrm{P}<0.05$ was considered to indicate a statistically significant difference.

\section{Results}

Apocynin treatment reduces upregulation of $\mathrm{NOX}_{2}$ protein expression. $\mathrm{NOX}_{2}$ protein expression was analyzed by western blot analysis (Fig. 1A). NOX 2 protein expression was identified at low levels in the CA1 region of the hippocampus in the sham group. Levels were markedly increased at 48 and $72 \mathrm{~h}$ 
A
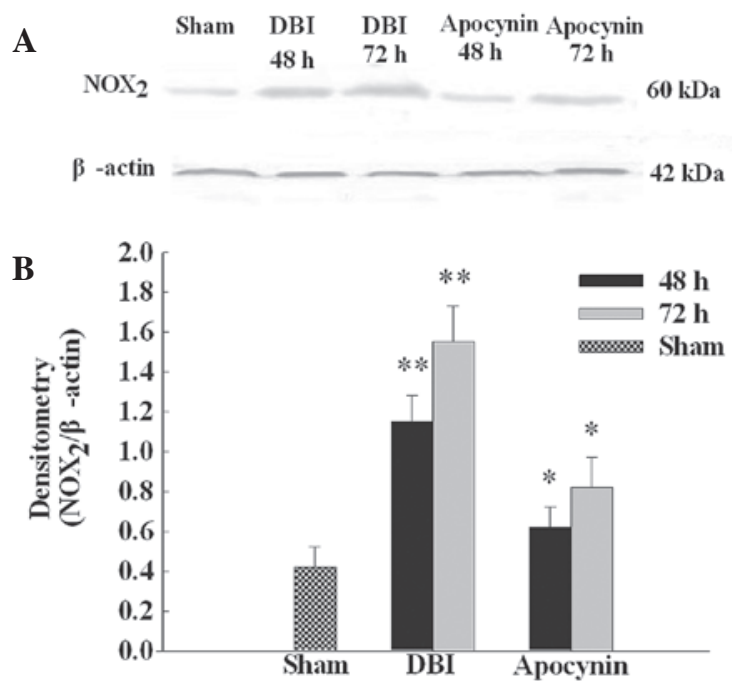

Figure 1. (A) Western blot analysis of $\mathrm{NOX}_{2}$ bands in the CA1 subregion of the hippocampus and (B) densitometry analysis of $\mathrm{NOX}_{2}$ bands corresponding to $\beta$-actin. Quantitative results of $\mathrm{NOX}_{2}$ protein are expressed as the mean of ratio of densitometries of $\mathrm{NOX}_{2}$ to $\beta$-actin bands \pm standard error for 5 experiments. Results demonstrated that $\mathrm{NOX}_{2}$ protein increased markedly at 48 and $72 \mathrm{~h}$ following DBI induction ( ${ }^{* *} \mathrm{P}<0.01 \mathrm{vs}$. sham group). Treatment with apocynin significantly decreased $\mathrm{NOX}_{2}$ protein expression ( $\mathrm{P}<0.05$, vs. DBI group). $\mathrm{NOX}_{2}$, catalytic subunit of nicotinamide adenine dinucleotide phosphate oxidase; DBI, diffuse brain injury.

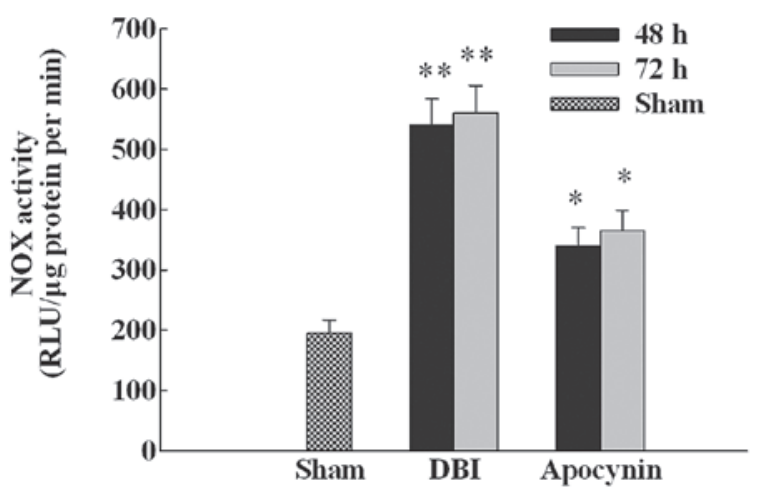

Figure 2. Effect of apocynin on NOX activity in the hippocampal CA1 subregion. Data are expressed as the means \pm standard error $(n=5)$. Activity of NOX in the CA1 subregions was markably enhanced at 48 and $72 \mathrm{~h}$ following DBI induction ("* $\mathrm{P}<0.01$, vs. sham group). Apocynin treatment reduced NOX activity ("P<0.05, vs. DBI group). NOX, nicotinamide adenine dinucleotide phosphate oxidase; DBI, diffuse brain injury.

following DBI induction. As demonstrated in Fig. 1B, $\mathrm{NOX}_{2}$ protein band intensity was quantified and results demonstrated that apocynin pre-treatment significantly inhibited the upregulation of $\mathrm{NOX}_{2}$ protein levels compared with the DBI groups.

Apocynin treatment depresses NOX activity. Since apocynin was found to inhibit the increased protein expression of $\mathrm{NOX}_{2}$ in the CA1 subregion of the hippocampus following DBI induction, we then performed a colorimetric assay to determine whether apocynin treatment reduces NOX activity. As demonstrated in Fig. 2, a marked elevation of NOX activity in the CA1 region was observed at 48 and $72 \mathrm{~h}$ in the DBI untreated group following DBI induction compared with the sham group. Apocynin treatment significantly attenuated NOX activity in

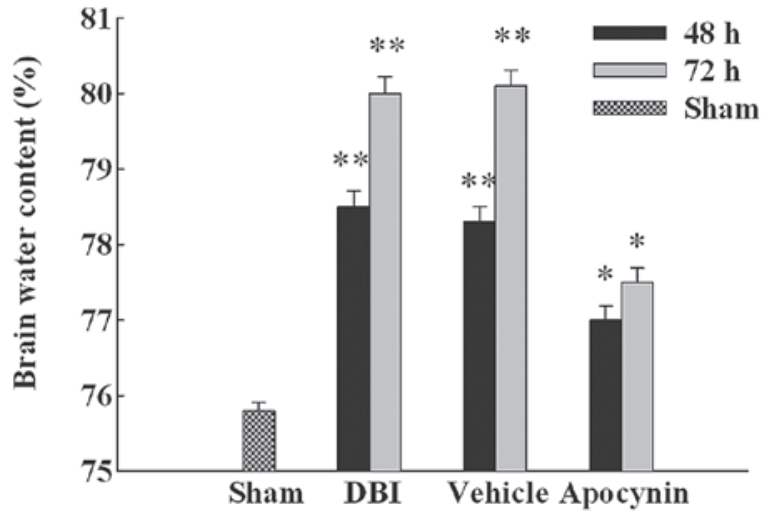

Figure 3. Effect of apocynin on brain edema. Brain water content was determined at 48 and $72 \mathrm{~h}$ following DBI and calculated as percentage of dry and wet ratio. Bars represent mean \pm standard error ( $n=5$ per group). Brain water content increased markedly at 48 and $72 \mathrm{~h}$ following DBI $\left({ }^{* *} \mathrm{P}<0.01\right.$, vs. sham group). Treatment with apocynin decreased brain edema ( $\mathrm{P}<0.05$, vs. DBI alone or DBI treated with vehicle groups), as demonstrated by a decrease in brain water content. DBI, diffuse brain injury.

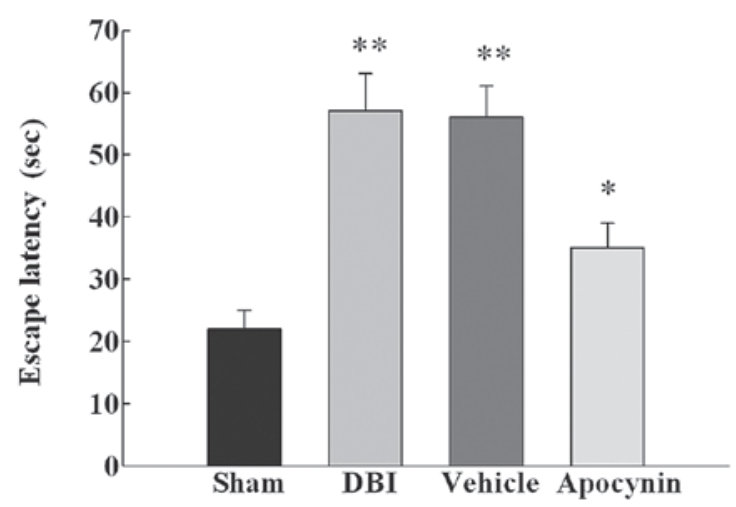

Figure 4. Effect of apocynin on escape latency performance in the Morris water maze at $72 \mathrm{~h}$ following DBI or sham surgery. Bars represent the mean \pm standard error ( $n=5$, per group). The escape latency increased markedly at 48 and $72 \mathrm{~h}$ following DBI $\left({ }^{* *} \mathrm{P}<0.01\right.$, vs. sham group). Treatment with apocynin reduced the time to identify the platform $\left({ }^{*} \mathrm{P}<0.01\right.$, vs. DBI alone or DBI treated with vehicle groups). DBI, diffuse brain injury.

the treated group compared with the DBI untreated or the DBI group treated with the vehicle; however, NOX activity levels remained higher than the sham group.

Apocynin treatment attenuates brain edema. The wet-dry method was used to evaluate brain edema. In order to examine whether brain edema is associated with the upregulation of $\mathrm{NOX}_{2}$ and NOX activation, we used apocynin, a specific inhibitor of NOX, as pre-treatment prior to DBI. As demonstrated in Fig. 3, DBI induced a significant increase in brain edema at 48 and $72 \mathrm{~h}$ in the DBI group compared with sham control. Pre-treatment with apocynin significantly reduced brain edema following DBI induction.

Apocynin treatment improves learning and memory ability. Since apocynin treatment was found to inhibit NOX activation and attenuate brain edema, we then examined whether apocynin treatment can improve spatial learning function using a Morris water maze $72 \mathrm{~h}$ following DBI induction or sham surgery. As demonstrated in Fig. 4, DBI caused a significant deficit in 
spacial learning at $72 \mathrm{~h}$ following DBI induction in the DBI groups compared with the sham group. Apocynin treatment reduced the escape latency in the treated group compared with DBI untreated group or the DBI group treated with the vehicle.

\section{Discussion}

Secondary damage following primary DBI leads to brain edema and neuronal cell death and aggravates neurological disfunctions. Effective management is imperative for promoting anatomical and functional recovery. NOX is a major complex that produces reactive ROS and has previously been associated with secondary damage, leading to secondary brain injury, following brain ischemia reperfusion (16) and cerebral ischemia stroke $(17,18)$. However, the role of NOX in the aggravation of brain damage following DBI in the rat model remains poorly understood.

In the present study, we confirmed that $\mathrm{NOX}_{2}$ protein expression and NOX activity were enhanced at 48 and $72 \mathrm{~h}$ following DBI induction. These observations were accompanied by an increase in brain water content and profound neurological dysfunction. Treatment with apocynin not only reduced upregulated $\mathrm{NOX}_{2}$ protein expression and NOX activity, but markedly attenuated brain edema and spatial learning deficits, as determined by Morris water maze performance. These results indicate that following DBI, NOX activation is pivotal in the additional aggravation of secondary brain damage.

Elucidation of the molecular mechanisms of brain edema is an important area of investigation, with the ultimate aim to develop new therapeutic interventions for the prevention of edema formation following traumatic brain injury. $\mathrm{NOX}_{2}$, a membrane catalytic subunit of NOX, has been demonstrated to be upregulated in the ischemic period (19). Previous studies using $\mathrm{NOX}_{2}$ mutant knockout mice or pre-treatment with the specific $\mathrm{NOX}_{2}$ inhibitor, gp91ds-tat, have demonstrated significantly attenuated neuronal damage and edema following traumatic brain injury (20-22). Dohi et al (20) and Lo et al (23) found that the inhibition of NOX activity improved neurological functions following surgically-induced brain injury or traumatic brain injury using transgenic mice lacking the $\mathrm{NOX}_{2}$ subunit of NOX. These studies all indicate that the beneficial neuroprotective effects of apocynin are specifically due to the inhibition of $\mathrm{NOX}_{2}$ expression. Consistent with these previous studies, the results of the present study revealed that treatment with apocynin reduced enhanced $\mathrm{NOX}_{2}$ expression and attenuated brain edema at 48 and $72 \mathrm{~h}$ following DBI induction. In addition, cognitive impairment was improved by apocynin treatment, which may be mediated, in part, by the downregulation of $\mathrm{NOX}_{2}$ following DBI.

NOX activity has previously been demonstrated to increase during brain injury following experimental ischemia (24). The activation of NOX is mediated by the translocation of cytosolic subunits to the cell membrane and fusion with $\mathrm{NOX}_{2}$. The active complex transfers electrons to oxygen, producing superoxide anions, a precursor of ROS $(1,25)$. The neuroprotective effects of apocynin have been hypothesized to be achieved by the downregulation of $\mathrm{NOX}_{2}$ expression and NOX activity. These events may depress the reduction of ROS (26), attenuating the permeability of the blood-brain barrier and further reducing brain edema following DBI. Alternative mechanisms of NOX activity associated with brain damage, including inflammation (27) and neuronal death (28) i.e., apoptosis (29) and autophagy cell death (30) should be investigated further in order to evaluate the detailed role of NOX in secondary brain damage following DBI.

In conclusion, the results of the present study demonstrate that the upregulation of $\mathrm{NOX}_{2}$ expression and NOX activation are involved in secondary brain damage following DBI. Pre-treatment with apocynin attenuates brain edema and improves spatial learning ability. This neuroprotection is associated with the blockage of NOX activity. In addition, the present study indicates that targeting NOX with specific NOX inhibitors may have clinical efficacy in DBI.

\section{Acknowledgements}

The present study was supported by a grant from the Natural Science Foundation of Hebei Province (no. H2012401071).

\section{References}

1. Bedard K and Krause KH: The NOX family of ROS-generating NADPH oxidases: physiology and pathophysiology. Physiol Rev 87: 245-313, 2007.

2. Infanger DW, Sharma RV and Davisson RL: NADPH oxidases of the brain: distribution, regulation and function. Antioxid Redox Signal 8: 1583-1596, 2006.

3. Kim MJ, Shin KS, Chung YB, Jung KW, Cha CI and Shin DH: Immunohistochemical study of p47Phox and gp91Phox distributions in rat brain. Brain Res 1040: 178-186, 2005.

4. Sorce $\mathrm{S}$ and Krause KH: NOX enzymes in the central nervous system: from signaling to disease. Antioxid Redox Signal 11: 2481-2504, 2009.

5. Serrano F, Kolluri NS, Wientjes FB, Card JP and Klann E: NADPH oxidase immunoreactivity in the mouse brain. Brain Res 24: 193-198, 2003.

6. Lafeber FP, Beukelman CJ, van den Worm E, et al: Apocynin, a plant-derived, cartilage-saving drug, might be useful in the treatment of rheumatoid arthritis. Rheumatology (Oxford) 38: 1088-1093, 1999.

7. Zhang Y, Chan MM andrews MC, et al: Apocynin but not allopurinol prevents and reverses adrenocorticotropic hormoneinduced hypertension in the rat. Am J Hypertens 18: 910-916, 2005.

8. Sawauchi S, Marmarou A, Beaumont A, Tomita Y and Fukui S: A new rat model of diffuse brain injury associated with acute subdural hematoma: assessment of varying hematoma volume, insult severity and the presence of hypoxemia. J Neurotrauma 20: 613-622, 2003.

9. Kahles T, Luedike P, Endres M, et al: NADPH oxidase plays a central role in blood-brain barrier damage in experimental stroke. Stroke 38: 3000-3006, 2007.

10. Tang LL, Ye K, Yang XF and Zheng JS: Apocynin attenuates cerebral infarction after transient focal ischaemia in rats. J Int Med Res 35: 517-522, 2007.

11. Connell BJ, Saleh MC, Khan BV and Saleh TM: Apocynin may limit total cell death following cerebral ischemia and reperfusion by enhancing apoptosis. Food Chem Toxicol 49: 3063-3069, 2011.

12. Murillo MM, Carmona-Cuenca I, Del Castillo G, et al: Activation of NADPH oxidase by transforming growth factor-beta in hepatocytes mediates up-regulation of epidermal growth factor receptor ligands through a nuclear factor-kappaB-dependent mechanism. Biochem J 405: 251-259, 2007.

13. Zhang QG, Raz L, Wang R, et al: Estrogen attenuates ischemic oxidative damage via an estrogen receptor alpha-mediated inhibition of NADPH oxidase activation. J Neurosci 29: 13823-13836, 2009.

14. Tang J, Liu J, Zhou C, et al: Mmp-9 deficiency enhances collagenase-induced intracerebral hemorrhage and brain injury in mutant mice. J Cereb Blood Flow Metab 24: 1133-1145, 2004.

15. Hui-guo L, Kui L, Yan-ning Z and Yong-jian X: Apocynin attenuate spatial learning deficits and oxidative responses to intermittent hypoxia. Sleep Med 11: 205-212, 2010. 
16. Woodfin A, Hu DE, Sarker M, Kurokawa T and Fraser P: Acute NADPH oxidase activation potentiates cerebrovascular permeability response to bradykinin in ischemia-reperfusion. Free Radic Biol Med 50: 518-524, 2011.

17. Yoshioka H, Niizuma K, Katsu M, et al: NADPH oxidase mediates striatal neuronal injury after transient global cerebral ischemia. J Cereb Blood Flow Metab 31: 868-880, 2011.

18. Kelly KA, Li X, Tan Z, VanGilder RL, Rosen CL and Huber JD: NOX2 inhibition with apocynin worsens stroke outcome in aged rats. Brain Res 1292: 165-172, 2009.

19. Hur J, Lee P, Kim MJ, Kim Y and Cho YW: Ischemia-activated microglia induces neuronal injury via activation of gp91phox NADPH oxidase. Biochem Biophys Res Commun 391: 1526-1530, 2010.

20. Dohi K, Ohtaki H, Nakamachi T, et al: Gp91phox (NOX2) in classically activated microglia exacerbates traumatic brain injury. J Neuroinflammation 7: 41, 2010.

21. Zhang QG, Laird MD, Han D, et al: Critical role of NADPH oxidase in neuronal oxidative damage and microglia activation following traumatic brain injury. PLoS One 7: e34504, 2012.

22. Jackman KA, Miller AA, De Silva TM, Crack PJ, Drummond GR and Sobey CG: Reduction of cerebral infarct volume by apocynin requires pretreatment and is absent in Nox2-deficient mice. Br J Pharmacol 156: 680-688, 2009.

23. Lo W, Bravo T, Jadhav V, Titova E, Zhang JH and Tang J: NADPH oxidase inhibition improves neurological outcomes in surgicallyinduced brain injury. Neurosci Lett 414: 228-232, 2007.
24. Vallet P, Charnay Y, Steger K, et al: Neuronal expression of the NADPH oxidase NOX4 and its regulation in mouse experimental brain ischemia. Neuroscience 132: 233-238, 2005.

25. Cairns B, Kim JY, Tang XN and Yenari MA: NOX inhibitors as a therapeutic strategy for stroke and neurodegenerative disease. Curr Drug Targets 13: 199-206, 2012.

26. Zia MT, Csiszar A, Labinskyy N, et al: Oxidative-nitrosative stress in a rabbit pup model of germinal matrix hemorrhage: role of NAD(P)H oxidase. Stroke 40: 2191-2198, 2009.

27. Mander PK, Jekabsone A and Brown GC: Microglia proliferation is regulated by hydrogen peroxide from NADPH oxidase. J Immunol 176: 1046-1052, 2006.

28. Jana A and Pahan K: Fibrillar amyloid-beta peptides kill human primary neurons via NADPH oxidase-mediated activation of neutral sphingomyelinase. Implications for Alzheimer's disease. J Biol Chem 279: 51451-51459, 2004

29. Bobba A, Atlante A, Petragallo VA and Marra E: Different sources of reactive oxygen species contribute to low potassiuminduced apoptosis in cerebellar granule cells. Int J Mol Med 21: 737-745, 2008

30. Luo CL, Li BX, Li QQ, et al: Autophagy is involved in traumatic brain injury-induced cell death and contributes to functional outcome deficits in mice. Neuroscience 184: 54-63, 2011. 